\title{
Chemo-resistant Gastric Cancer Associated Gene Expression Signature: Bioinformatics Analysis Based on Gene Expression Omnibus
}

\author{
JUN-BAO LIU ${ }^{*}$, TUNYU JIAN ${ }^{2 *}$, CHAO YUE ${ }^{3}$, DAN CHEN $^{4}$, WEI CHEN ${ }^{5}$, TING-TING BAO ${ }^{6}$, HAI-XIA LIU ${ }^{7}$, \\ YUN CAO ${ }^{8}$, WEI-BING LI $^{6}$, ZHIJIAN YANG ${ }^{9}$, ROBERT M. HOFFMAN ${ }^{9}$ and CHEN YU ${ }^{6}$ \\ ${ }^{1}$ Traditional Chinese Medicine Department, People's Hospital of Henan Province, \\ People's Hospital of Zhengzhou University, Zhengzhou, P.R. China; \\ ${ }^{2}$ Institute of Botany, Jiangsu Province and Chinese Academy of Sciences, Nanjing, P.R. China; \\ ${ }^{3}$ Department of general surgery, Jiangsu Cancer Hospital \& Jiangsu Institute of Cancer Research \& \\ The Affiliated Cancer Hospital of Nanjing Medical University, Nanjing, P.R. China; \\ ${ }^{4}$ Research Center of Clinical Oncology, Jiangsu Cancer Hospital \& Jiangsu Institute of Cancer Research \& \\ The Affiliated Cancer Hospital of Nanjing Medical University, Nanjing, P.R. China; \\ ${ }^{5}$ Department of Head and Neck Surgery, Jiangsu Cancer Hospital \& Jiangsu Institute of Cancer Research \& \\ The Affiliated Cancer Hospital of Nanjing Medical University, Nanjing, P.R. China; \\ ${ }^{6}$ Department of Integrated TCM \& Western Medicine, Jiangsu Cancer Hospital \& \\ Jiangsu Institute of Cancer Research \& The Affiliated Cancer Hospital of Nanjing \\ Medical University, Nanjing, P.R. China; \\ ${ }^{7}$ Emergency Department, The Second Affiliated Hospital of Nanjing \\ University of Chinese Medicine, Nanjing, P.R. China; \\ ${ }^{8}$ Master candidate of Oncology, Nanjing University of Chinese Medicine, Nanjing, P.R. China; \\ ${ }^{9}$ AntiCancer, Inc., San Diego, CA, U.S.A.
}

\begin{abstract}
Background/Aim: This study aimed to identify biomarkers for predicting the prognosis of advanced gastric cancer patients who received docetaxel, cisplatin, and $S-1$ (DCS). Materials and Methods: Gene expression profiles were obtained from the Gene Expression Omnibus database (GSE31811). Gene-Ontology-enrichment and KEGG-pathway analysis were used for evaluating the biological functions of differentially-expressed genes. Protein-protein interaction (PPI) network and Kaplan-Meier survival analyses were employed to assess the prognostic values of hub genes. Results: A total of 1,486 differentially expressed genes (DEGs) were
\end{abstract}

This article is freely accessible online.

\footnotetext{
*These Authors contributed equally to this work.

Correspondence to: Chen Yu, Department of Integrated TCM \& Western Medicine, Jiangsu Cancer Hospital \& Jiangsu Institute of Cancer Research \& The Affiliated Cancer Hospital of Nanjing Medical University, Nanjing, Jiang Su, 210000, P.R. China. Tel: +86 15850569736, e-mail: 41186709@qq.com
}

Key Words: Dysregulated genes, gene ontology, KEGG pathway, gastric cancer, protein-protein interaction, chemoresistance. identified, including 13 up-regulated and 1,473 down-regulated genes. KEGG pathways such as metabolic pathways, cell adhesion molecules (CAMs), PI3K-Akt signaling pathway and pathways in cancer were significantly represented. In the PPI network, the top ten hub genes ranked by degree were GNG7, PLCB1, CALML5, FGFR4, GRB2, JAK3, ADCY7, ADCY9, GNAS and KDR. Five DEGs, including ANTXR1, EFNA5, GAMT, E2F2 and NRCAM, were associated with relapse-free survival and overall survival. Conclusion: ANTXR1, EFNA5, GAMT, E2F2 and NRCAM are potential biomarkers and therapeutic targets for DCS treatment in GC.

Gastric cancer (GC), as the fourth most common cancer, has become a major global heath challenge. It is estimated that about 934,000 new GC cases are diagnosed and that there are about 700,000 mortalities annually (1). In China, GC is estimated to cause more than 220,000 mortalities annually, which accounts for approximately one-third of global gastric cancer mortalities (2). Despite improvement in diagnosis and treatment, the prognosis of gastric cancer patients remains very poor. Surgery is the primary treatment for early-stage GC (3). Chemotherapy plays an important role in GC treatment since most gastric cancers are initially diagnosed at advanced or metastatic stages (4). The efficacy of a triple-drug combination 
chemotherapy regimen consisting of docetaxel, cisplatin, and S1 (DCS) has been investigated for the treatment of advanced GC. DCS treatment was reported to be well tolerated in metastatic GC patients, with a very high response rate $(87.1 \%)$, and a high downstaging rate $(25.8 \%)$ leading to a high curative surgery rate $(22.6 \%)$ (5-6). Furthermore, in a Phase II study of this regimen showed that DCS treatment for localized advanced GC (AGC) was feasible and highly effective with a $74.4 \%$ response rate, and disease control ratio was $100 \%$. In addition, DCS as neo-adjuvant treatment for localized AGC demonstrated a sufficient R0 resection rate and a good pathological response with manageable toxicities (7). However, efficiency of DCS regimen is limited by chemoresistance in some cancer patients. Biomarkers for prognosis prediction and their underlying molecular mechanisms are yet to be fully elucidated.

In this study, a set of differentially expressed genes (DEGs) were obtained from the microarray dataset GSE31811, which was downloaded from Gene Expression Omnibus (GEO; https://www.ncbi.nlm.nih.gov/geo/), containing 19 patients with unresectable metastatic GC who received DCS as first-line therapy. We identified potential biomarkers and pathways associated with DCS resistance by analyzing GO and KEGG pathway enrichment, and constructing a protein-protein interaction (PPI) network. The prognostic values of the biomarkers and their potential underlying mechanisms were also assessed.

\section{Materials and Methods}

Chemo-resistant gastric cancer patient datasets. Genome-wide gene expression profile data generated from the Affymetrix platform (Affymetrix Human Genome U133 Plus 2.0 Arrays) and the corresponding clinical information of patients with chemo-resistant GC were retrieved from the GEO database (https:// www.ncbi.nlm.nih.gov/geo). "chemo-resistant gastric cancer" was used as a keyword to perform queries. Inclusion criteria were as follows: species-Homo sapiens, expression profiling by array, and samples with available clinical information for analysis. The expression microarray datasets GSE31811 and GSE26253 were downloaded. Overall, 19 patients with unresectable metastatic gastric cancer who received DCS as first-line therapy were enrolled in this study. No approval was required from the ethics committee because these data were obtained using the GEO database. The workflow chart is shown in Figure 1.

Gene expression profile data. The GSE31811 dataset consisted of 19 patients with unresectable metastatic gastric cancer who received DCS as a first-line therapy. According to RECIST criteria, 8 patients were identified as non-responders and 11 patients as earlyresponders. The GSE26253 signature, that comprised 432 samples of gastric cancer patients that had undergone curative surgery plus chemoradiotherapy, was applied to verify the model.

DEGs identification. The differentially-expressed genes associated with DCS resistance were obtained from GSE31811. The mRNA expression profile was performed using an Agilent 2100 Bioanalyzer (Agilent Technologies, Palo Alto, CA, USA). The Benjamini and

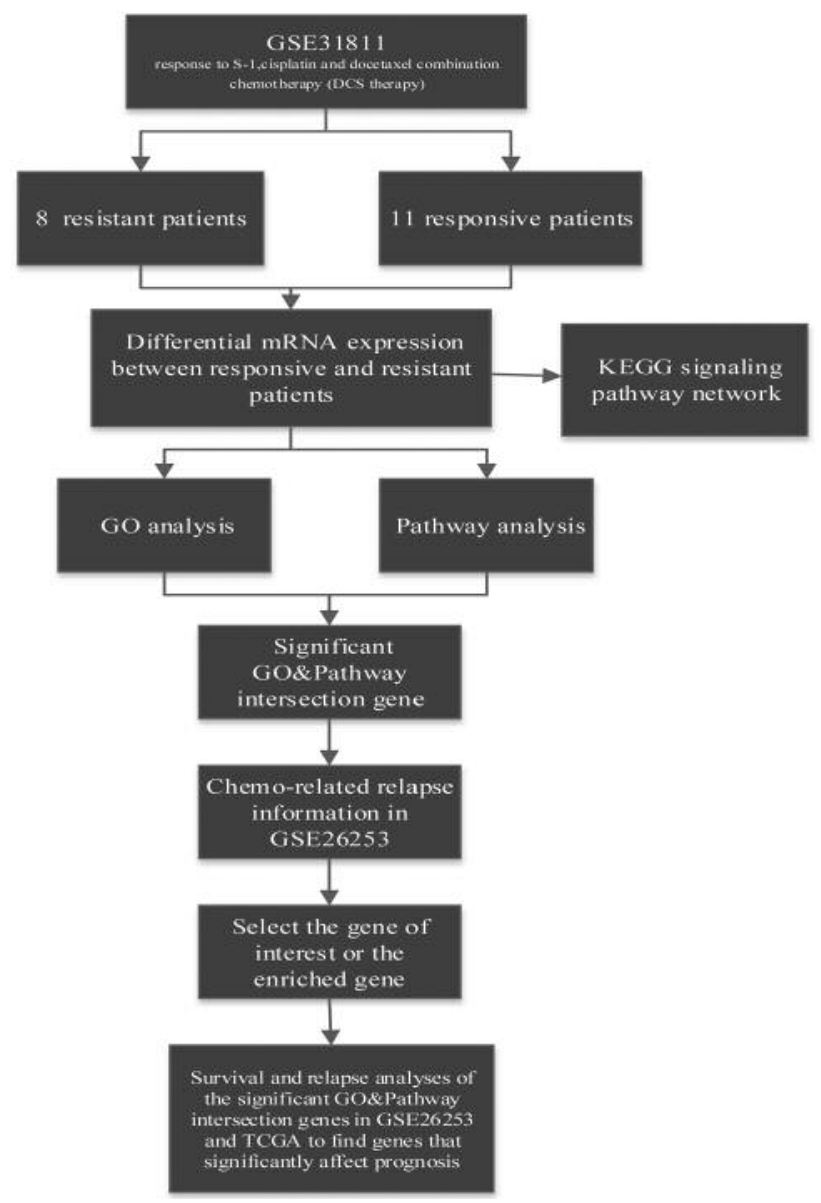

Figure 1. Bioinformatics flowchart of the GEO database.

Hochberg method was used to adjust false-positives. Fold-change (FC) in gene expression was calculated with the threshold criteria of the adj. $p<0.05$ and $\mid \log 2 \mathrm{FCl} \geq 1$ were set for DEGs selection. Then, we selected intersection mRNAs and samples with the survival information obtained from GSE26253 and TCGA for further analysis.

Gene ontology and pathway analysis. All DEGs were placed in the analysis tool Database for Annotation, Visualization and Integrated Discovery (DAVID, http://david.abcc.ncifcrf. gov/) to obtain relevant functional annotation. DAVID was employed for gene ontology (GO), which supplied information about molecular functions, cellular components, and biological processes. In addition, DAVID was also used for analyzing Kyoto Encyclopedia of Genes and Genomes (KEGG, http://www. kegg.jp/) pathway enrichment of DEGs.

Protein-protein interactions (PPI) network and module analysis. Cytoscape v3.0 software was used for constructing an mRNAmRNA interaction network, which was based on GO enrichment and pathway analysis of differentially- expressed genes. The matrix of gene expression values was visualized graphically. The geneinteraction relationship was represented by nodes and edges in the graph. The types of gene-gene interaction relationships included 
Diff Up Gene GO-Analysis

Negative regulation of MAP kinase activity Positive regulation of myosin light chain

Positive regulation of $\mathrm{T}$ cell mediated Negative regulation of glucose transport Sequestering of triglyceride Negative regulation of lipid metabolic Positive regulation of hydrolase activity Negative regulation of meiotic cell cycle Positive regulation of cell adhesion Negative regulation of adiponectin Regulation of immune response Interleukin-1 beta production

Positive regulation of lipid catabolic Interleukin-6 production

Regulation of mitotic cell cycle spindle Ectopic germ cell programmed cell death Positive regulation of chemokine Positive regulation of interleukin-6 Glycine transport Positive regulation of histone

\section{Diff Down Gene GO-Analysis}

Signal transduction Intracellular signal transduction Positive regulation of apoptotic process Transcription from RNA polymerase II promoter Cell differentiation Inflammatory response Antigen processing and presentation of exogenous Negative regulation of cell proliferation Regulation of transcription from RNA polymerase Protein ubiquitination MAPK cascade Innate immune response Positive regulation of cell proliferation Post-translational protein modification Protein phosphorylation Immune response T cell receptor signaling pathway Regulation of receptor activity Apoptotic process Regulation of apoptotic process

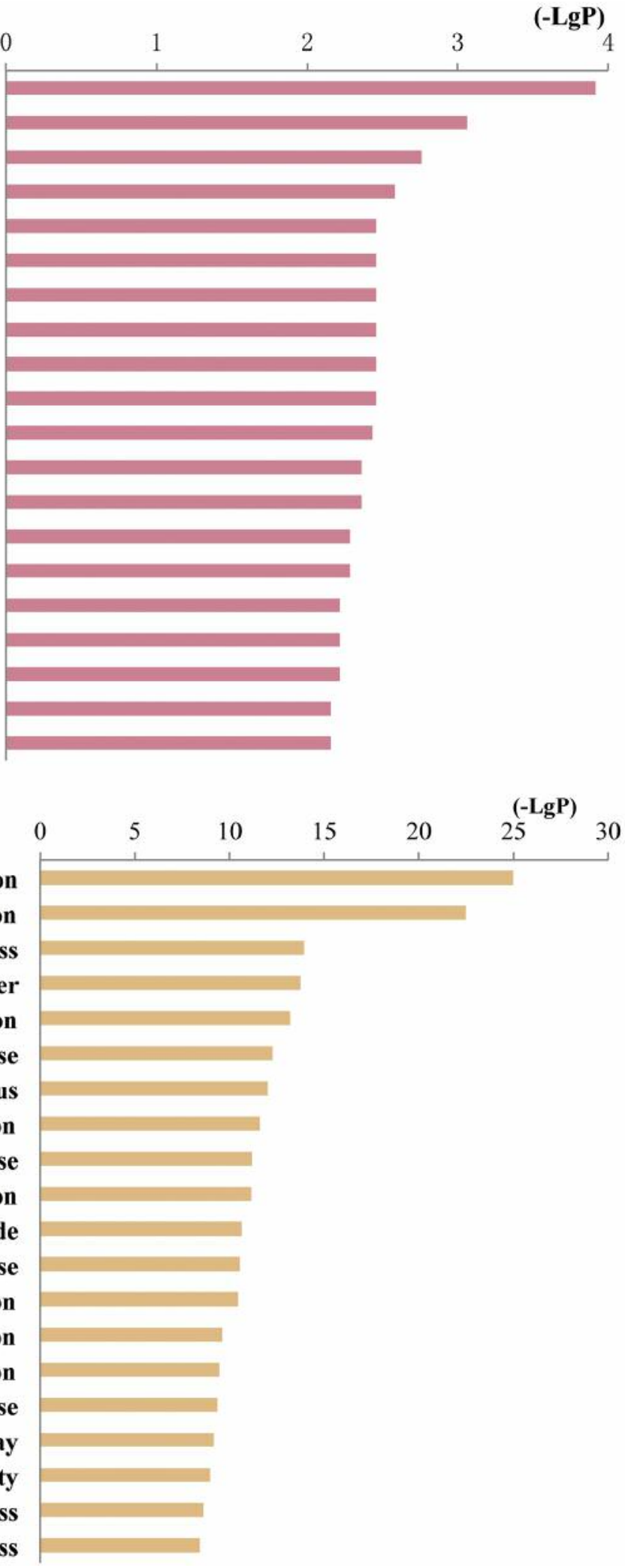

Figure 2. Top 20 enriched GO terms for differentially up-regulated and down-regulated genes (the bar plot presents the enrichment scores of the significantly enriched GO terms). 
Diff Up Gene Pathway-Analysis

Fluid shear stress and atherosclerosis

NOD-like receptor signaling pathway

MAPK signaling pathway

Ascorbate and aldarate metabolism

Antifolate resistance

Pentose and glucuronate interconversions

Prion diseases

African trypanosomiasis

Fat digestion and absorption

Graft-versus-host disease

Porphyrin and chlorophyll metabolism

Type I diabetes mellitus

Drug metabolism - other enzymes

Malaria

Legionellosis

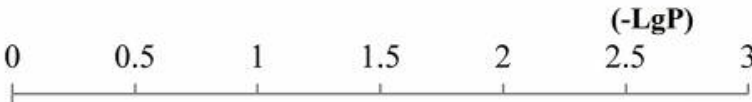

\footnotetext{
\begin{tabular}{|}
\hline \\
\hline \\
\hline
\end{tabular}
}
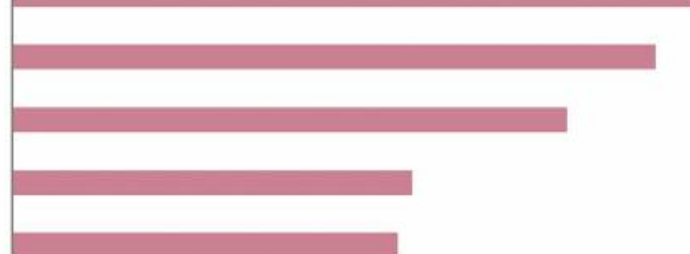

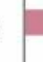

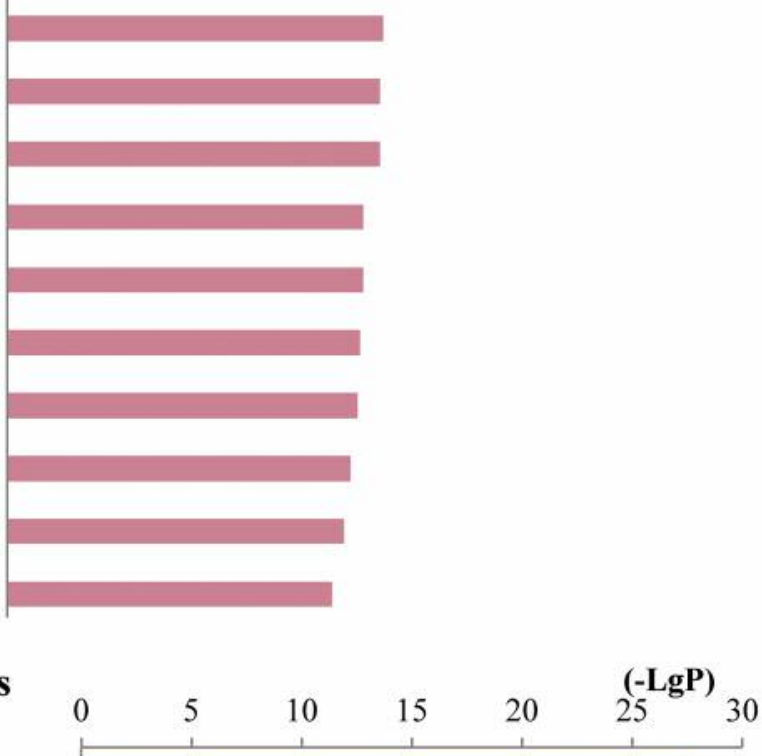

Metabolic pathways

Cell adhesion molecules (CAMs)

PI3K-Akt signaling pathway

Pathways in cancer

Regulation of actin cytoskeleton

Ras signaling pathway

Apelin signaling pathway

Chemokine signaling pathway

Intestinal immune network for IgA production

Rap1 signaling pathway

Autophagy - animal

MAPK signaling pathway

Signaling pathways regulating pluripotency of

EGFR tyrosine kinase inhibitor resistance

ErbB signaling pathway

Figure 3. Top 15 enriched KEGG pathways for differentially up-regulated and down-regulated genes (the bar plot presents the enrichment scores of the significantly enriched KEGG pathways). 


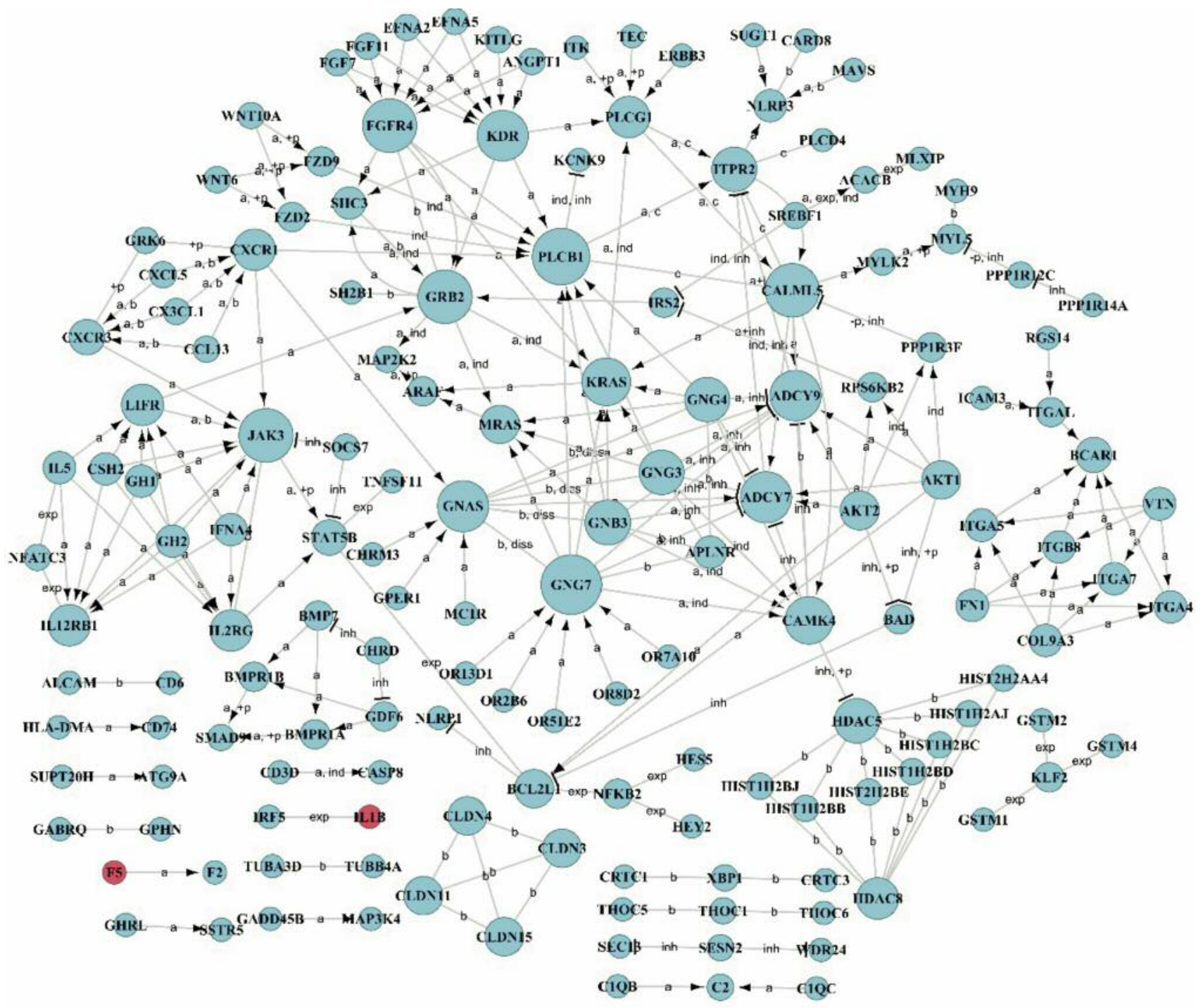

Figure 4. Signal network of differentially expressed genes. Red cycle nodes represent up-regulated genes, and green cycle nodes represent downregulated genes. Edges represent interactions between genes (arrowheads represent targets). Interaction types: a: activation; $b$ : binding/association; c: compound; exp: expression; ind: indirect effect; inh: inhibition; +p: phosphorylation; - p: dephosphorylation.

phosphorylation, dephosphorylation, inhibition and activation. In the signaling network, the size of the cycle was considered as the frequency of the gene interaction. The most prominent central genes in the network indicated the genes with the highest frequency.

Evaluation of prognostic value of hub genes. Kaplan-Meier (KM) plots enable comprehensive analysis of prognostic values among lists of genes in GC based on GSE26253 and TCGA. The prognostic value of hub genes for relapse-free survival (RFS) were displayed with the hazard ratios (HR) and log-rank $p$-values. To identify potential candidate biomarkers for predicting overall survival (OS) of GC patients, the gene expression profiling interactive analysis (GEPIA, http://gepia.cancer-pku.cn) was established for customized genomic analysis based on the Cancer Genome Atlas (TCGA) database.
Statistical analysis. Generally, a $p$-value $<0.05$ was defined as a cutoff criterion and considered statistically significant in all cases. SPSS 17.0 (Chicago, IL, USA) and Prism 5.0 (GraphPad Software, San Diego, CA, USA) were used for statistical analysis and illustration.

\section{Results}

GO enrichment analysis of DEGs. A total of 1,486 DEGs were identified to be associated with DCS-resistance, with 13 genes up-regulated and 1473 down-regulated. GO enrichment results were conducted by uploading all DEGs to the online tool DAVID (http://david.abcc.ncifcrf.gov/). First, the down-regulated genes were analyzed. This study 

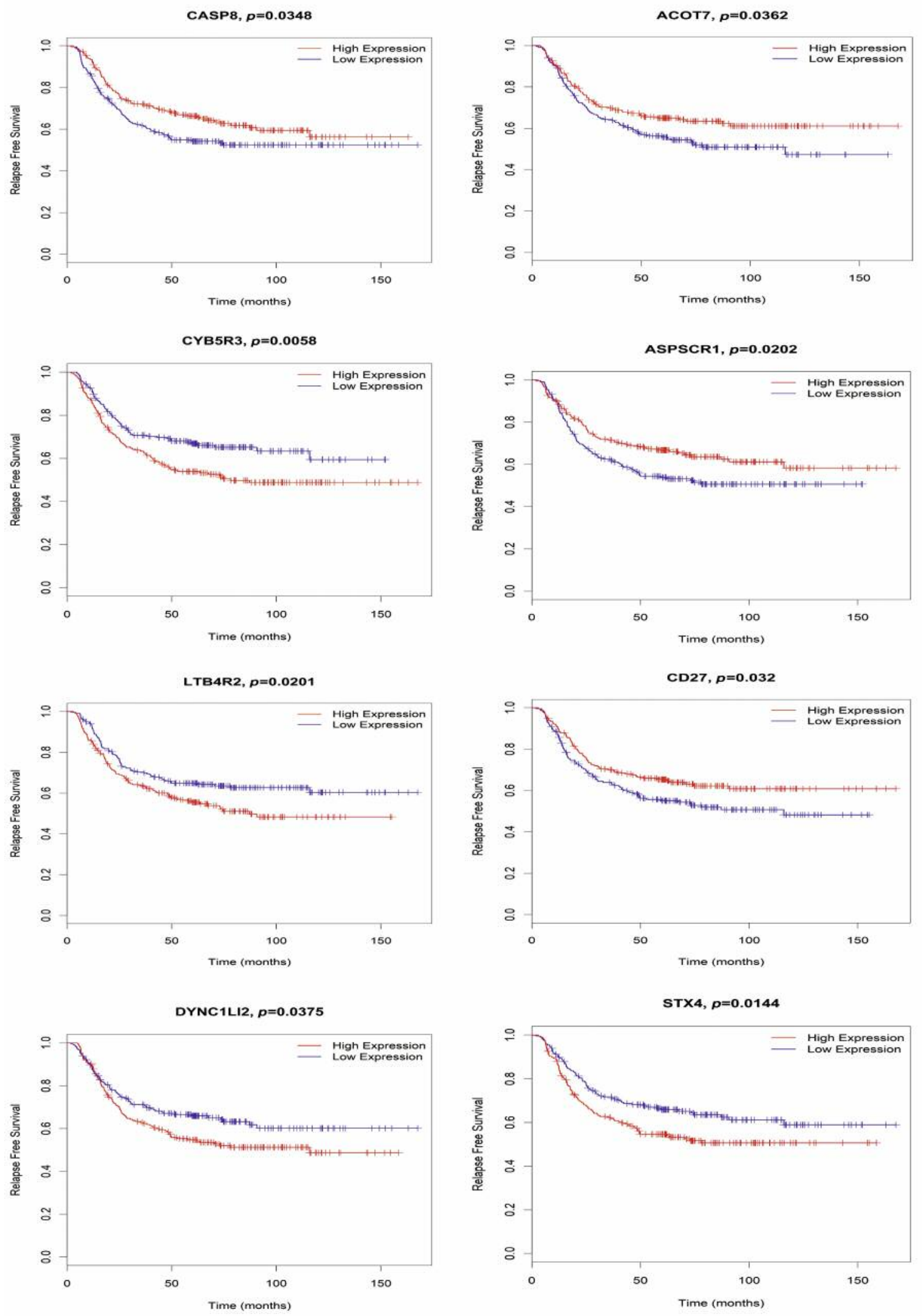

Figure 5. Continued 

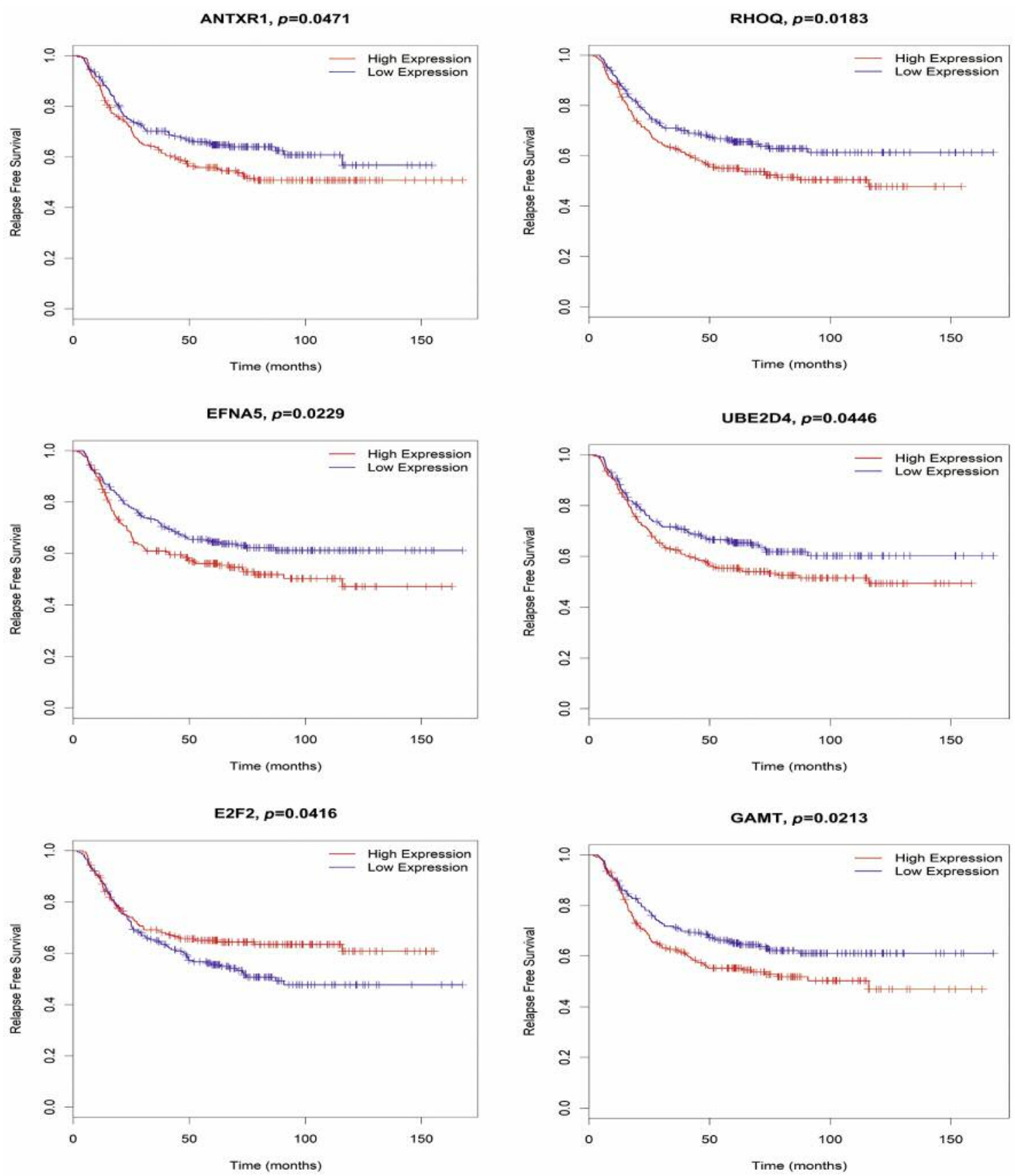

Figure 5. Kaplan-Meier survival curves for 5 genes whose expression is associated with overall survival. Vertical axis: Probability of overall survival; Horizontal axis: survival years.

found that the expression of 1,473 genes was decreased in GC patients who were DCS-resistant compared with DCSsensitive patients. Most of the down-regulated genes were involved in biological processes such as signal transduction, intracellular signal transduction, apoptosis and inflammatory response. Then, 13 up-regulated DEGs were used for GO-enrichment analysis. The results showed that up-regulated DEGs were primarily involved in negative regulation of MAP kinase activity, positive regulation of myosin light chain kinase activity, positive regulation of $\mathrm{T}$ cell mediated immunity, negative regulation of glucose transport and sequestering of triglycerides (Figure 2). GO-enrichment analysis suggested that the differentially-expressed mRNAs may be involved in the regulation of gene transcription, cell apoptosis and cellular inflammatory responses. 

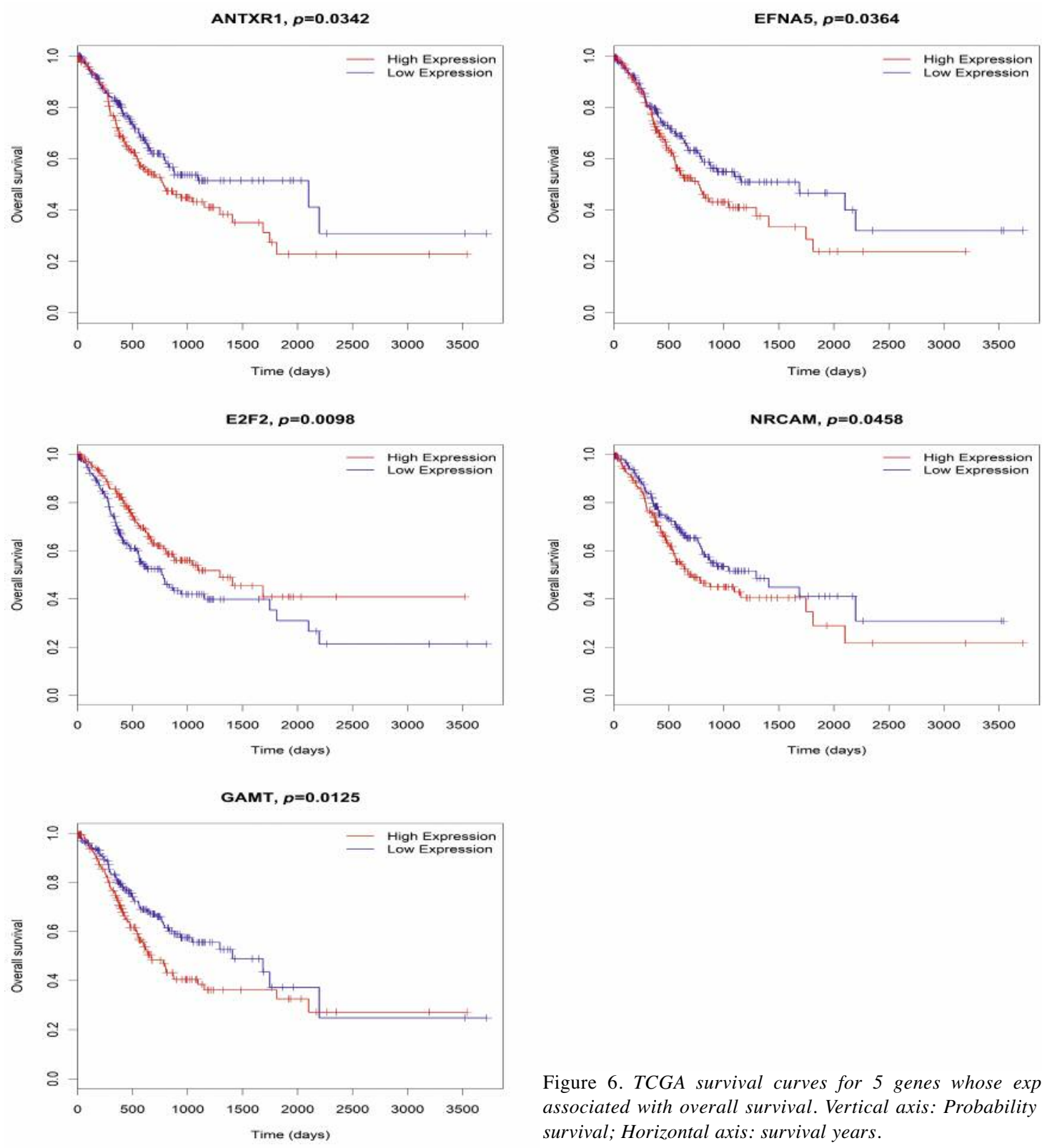

Figure 6. TCGA survival curves for 5 genes whose expression is associated with overall survival. Vertical axis: Probability of overall survival; Horizontal axis: survival years.

KEGG pathway analysis of DEGs. The KEGG-pathway program (http://www.genome.jp/kegg/tool/search pathway.html) was used to verify the signaling pathways corresponding to the up-regulated genes and the down-regulated genes, respectively. Pathway analysis demonstrated that the significant pathways corresponding to the up-regulated genes were fluid shear stress and atherosclerosis, the NOD-like receptor signaling pathway, MAPK signaling pathway, antifolate resistance and ascorbate metabolism. Moreover, down-regulated DEGs were significantly enriched in metabolic pathways, cell-adhesion molecules (CAMs), the PI3K-Akt signaling pathway and pathways involved in cancer. Among those enriched pathways,

DGEs included those in the MAPK signaling pathway and PI3K-AKT signaling pathway which have been demonstrated to be associated with chemoresistance, invasion, and metastasis (8-10). "Pathways in cancer" that regulated the proliferation and apoptosis of cancer cells has been verified (11). KEGG pathway analysis indicated that some of the classic pathways involved in chemoresistance of GC were activated (Figure 3).

PPI network and module analysis. The PPI network of DEGs was constructed with Cytoscape v3.0 software based on GO and KEGG pathway analysis. The top ten genes ranked by degree were identified as hub genes. Hub genes including GNG7, 
PLCB1, CALML5, FGFR4, GRB2, JAK3, ADCY7, ADCY9, GNAS and KDR had the highest degree of nodes among the hub genes. In addition, high expression of the chemokine family of proteins, such as GRB2 and JAK3, involved in proliferation, migration, and invasion of cancers, was found $(12,13)$. Moreover, FGFR4, reported to be associated with epithelialmesenchymal transition (EMT) and metastasis, was also shown to be significant in the PPI network $(14,15)$. The up-regulated and down-regulated genes had complex interactions with other genes in the signaling network (Figure 4).

Prognostic analysis of hub genes. Furthermore, the mRNA expression of the hub genes was externally validated in GSE26253. KM-plot analysis was used to elucidate the influence of the DGEs in GSE26253 on RFS. A total of 34 DEGs were associated with RFS. Increased expression of 9 genes, ANTXR1, CYB5R3, DYNC1LI2, EFNA5, GAMT, LTB4R2, RHOQ, STX4 and UBE2D4, was associated with a decreased RFS rate. The increased expression of 25 genes was associated with an increased RFS rate, including E2F2, ACOT7, ASPSCR1, CASP8 and CD27 (Figure 5). Moreover, among the above 34 genes, increased expression of four genes (ANTXR1, EFNA5, GAMT and NRCAM) was associated with a decreased OS rate. In addition, increased expression of E2F2 was associated with an increased OS rate (Figure 6).

\section{Discussion}

Metastasis is responsible for the poor prognosis of advanced GC patients (16-17). DCS treatment was reported to be well tolerated and highly effective in patients with metastatic GC (5-6). However, DCS chemoresistance remains one of the major challenges in the treatment of metastatic GC. Therefore, identification of key genes and potential mechanisms underlying DCS resistance should improve prognosis of patients treated with DCS treatment.

The GSE19069 dataset was used in the present study for a comprehensive bioinformatics analysis and 13 up-regulated and 1473 down-regulated DEGs were identified. The results of the GO analysis demonstrated that the down-regulated DEGs were significantly related to apoptosis and development, while the up-regulated DEGs were related to cell proliferation and immune response. The KEGG enrichment analysis indicates that the DEGS were significantly involved in metabolic pathways and the PI3K-Akt signaling pathway.

Metabolic pathways are crucial for the growth and survival of cancer cells. Cancer cells require high energy and metabolic pathways are modified to match this need. The biosynthesis of molecules and the generation of energy by metabolic pathways could protect cancer cells from oxidative stress and compensate for a reduced supply of oxygen and nutrients. Thus, metabolic pathways support cancer cell proliferation, angiogenesis, metastasis and prevent apoptosis
(18-20). Our results suggest that metabolic reprogramming may be the cause of DCS-resistance found in GC patients.

The PI3K/Akt signaling pathway regulates cancer cell transcription, translation, proliferation, growth, and survival (21). Moreover, deregulation of the $\mathrm{PI} 3 \mathrm{~K} / \mathrm{Ak}$ pathway is frequently encountered in GC and plays an important role in tumor initiation and progression. The PI3K/Akt pathway was also reported to be associated with the metastatic cascade in GC, which includes cytoskeletal remodeling, proteolytic activity and resistance to chemotherapy. Oki et al.. revealed that the PI3K-Akt signaling pathway was correlated with increased resistance to multiple chemotherapeutic agents such as 5-fluorouracil (5-FU), doxorubicin, and cisplatin (22). Thus, the PI3K-Akt signaling pathway alterations may be biomarkers for predicting the efficacy of DCS therapy. Understanding the specific association between related DGEs expression and $\mathrm{PI} 3 \mathrm{~K} / \mathrm{Akt} / \mathrm{mTOR}$ pathway alterations in GC may lead to the development of new therapeutic strategies for DCS.

In PPI network analysis, we identified hub genes, which consisted of the ten DEGs with the highest degree of interaction. Noteworthy, seven of the 10 hub genes (GNAS, ADCY7, ADCY9, CALML5, FGFR4, KDR, PLCB1) associated with DCS resistance were commonly enriched in the Rap1 signaling pathway. Rap1 is a small GTPase and belongs to the Ras-related protein family. It plays a specific role in the regulation of many cellular processes, including cytoskeletal rearrangement and cell cycle progression (23, 24). RAP1 has also been linked to the control of cancer-cell growth and survival. Mechanistically, Ras was reported to initiate and sustain ERK signaling, which is activated in many malignancies and may be a target for cancer therapy (25).

Noteworthy, the present study was the first in silico analysis focusing on bioinformatics analysis of DCS resistance in GC, predicting the key genes and pathways associated with DCS resistance. In addition, the present study also investigated the prognostic values of key genes. Five DGEs, including ANTXR1, EFNA5, GAMT, E2F2 and NRCAM, were associated with DFS and OS.

In conclusion, our study revealed DCS-resistance-related genes and pathways in GC patients using bioinformatics. Gene expression of ANTXR1, EFNA5, GAMT, E2F2 and NRCAM were identified as potential prognosis markers for DCS chemotherapy response in GC patients.

\section{Conflicts of Interest}

The Authors declare no conflicts of interest regarding this study.

\section{Acknowledgements}

This study was supported by National Natural Science Foundation of China (No. 81503528), the Talents Program of Jiangsu Cancer Hospital and Financial Grant from the China Postdoctoral Science Foundation (No. 2018M632267). 


\section{Authors' Contributions}

Jun-bao Liu and Chao Yue collected clinical data; Dan Chen, Wei Chen and Tunyu Jian analyzed the data; Ting-Ting Bao, Hai-Xia Liu and Yun Cao perfomed statistical analysis. Wei-Bing Li wrote draft manuscript; Zhijian Yang and Robert M. Hoffman revised the manuscript.

\section{References}

1 Siegel RL, Miller KD and Jemal A: Cancer statistics, 2016. CA Cancer J Clin 66(1): 7-30, 2016. PMID: 26742998. DOI: $10.3322 /$ caac. 21332

2 Chen W, Zheng R, Baade PD, Zhang S, Zeng H, Bray F, Jemal $\mathrm{A}, \mathrm{Yu} \mathrm{XQ}$ and $\mathrm{He} \mathrm{J}$ : Cancer statistics in China, 2015. CA Cancer J Clin 66(2): 115-132, 2016. PMID: 26808342. DOI: 10.3322/ caac. 21338

3 Japanese Gastric Cancer Association: Japanese gastric cancer treatment guidelines 2014. Gastric Cancer 20(1): 1-19, 2017. PMID:15061149. DOI: 10.1007/s10120-016-0622-4

4 Webb A, Cunningham D, Scarffe JH, Harper P, Norman A and Joffe JK: Randomized trial comparing epirubicin, cisplatin, and fluorouracil versus fluorouracil, doxorubicin, and methotrexate in advanced esophagogastric cancer. J Clin Oncol 15(1): 261 267, 1997. PMID: 8996151. DOI: 10.1200/JCO.1997.15.1.261

5 Takayama T, Sato Y, Sagawa T, Okamoto T, Nagashima H and Takahashi Y: Phase I study of S-1, docetaxel and cisplatin combination chemotherapy in patients with unresectable metastatic gastric cancer. Br J Cancer 97(7): 851-856, 2007. PMID: 17848958. DOI: 10.1007/s00280-009-1215-2

6 Sato Y, Takayama T, Sagawa T, Takahashi Y, Ohnuma H and Okubo S: Phase II study of S-1, docetaxel and cisplatin combination chemotherapy in patients with unresectable metastatic gastric cancer. Cancer Chemother Pharmacol 66(4): 721-728, 2010. PMID: 20041328. DOI: 10.1007/s00280-0091215-2

7 Hirakawa M1, Sato Y, Ohnuma H, Takayama T, Sagawa T and Nobuoka T: A phase II study of neoadjuvant combination chemotherapy with docetaxel, cisplatin, and S-1 for locally advanced resectable gastric cancer: nucleotide excision repair (NER) as potential chemoresistance marker. Cancer Chemother Pharmacol 71(3): 789-797, 2013. PMID: 23338051. DOI: 10.1007/s00280-013-2073-5

8 Sumimoto H1, Imabayashi F, Iwata T and Kawakami Y: The BRAF-MAPK signaling pathway is essential for cancer-immune evasion in human melanoma cells. J Exp Med 203(7): 16511656, 2006. PMID: 16801397. DOI: 10.1084/jem.20051848

9 Beeram M1, Patnaik A and Rowinsky EK: Raf: a strategic target for therapeutic development against cancer. J Clin Oncol 23(27): 6771-6790, 2005. PMID: 16170185. DOI: 10.1200/jco.2005. 08.036

10 Byun WS, Jin M, Yu J, Kim WK, Song J and Chung HJ: A novel selenonucleoside suppresses tumor growth by targeting Skp2 degradation in paclitaxel-resistant prostate cancer. Biochem Pharmacol 158: 84-94, 2018. PMID: 30292755. DOI: 10.1016/ j.bcp.2018.10.002

11 Zhou LL, Xu XY, Ni J, Zhao X, Zhou JW and Feng JF: T-cell lymphomas associated gene expression signature: Bioinformatics analysis based on gene expression Omnibus. Eur J Haematol 100(6): 575-583, 2018. PMID: 29505095. DOI: 10.1111/ EJH.13051
12 Timsah Z, Ahmed Z, Ivan C, Berrout J, Gagea M and Zhou Y: Grb2 depletion under non-stimulated conditions inhibits PTEN, promotes Akt-induced tumor formation and contributes to poor prognosis in ovarian cancer. Oncogene 35(17): 2186-2196, 2016. PMID: 26212011. DOI: 10.1038/onc.2015.279

$13 \mathrm{Li} \mathrm{SD}, \mathrm{Ma} \mathrm{M}, \mathrm{Li} \mathrm{H}$, Waluszko A, Sidorenko T and Schadt EE: Cancer gene profiling in non-small cell lung cancers reveals activating mutations in JAK2 and JAK3 with therapeutic implications. Genome Med 9(1): 89, 2017. PMID: 29082853. DOI: $10.1186 / \mathrm{s} 13073-017-0478-1$

14 Prieto-Dominguez N, Shull AY and Teng Y: Making way for suppressing the FGF19/FGFR4 axis in cancer. Future Med Chem 10(20): 2457-2470, 2018. PMID: 30325210. DOI: $10.4155 / \mathrm{fmc}-$ 2018-0099

$15 \mathrm{Yu}$ T, Wang LN, Li W, Zuo QF, Li MM and Zou QM: Downregulation of miR-491-5p promotes gastric cancer metastasis by regulating SNAIL and FGFR4. Cancer Sci 109(5): 1393-1403, 2018. PMID: 29569792. DOI: 10.1111/cas.13583

16 Dahdaleh FS and Turaga KK: Evolving treatment strategies and outcomes in advanced gastric cancer with peritoneal metastasis. Surg Oncol Clin N Am 27(3): 519-537, 2018. PMID: 29935687. DOI: $10.1016 /$ j.soc.2018.02.006

17 Shimizu D, Kanda M and Kodera Y: Emerging evidence of the molecular landscape specific for hematogenous metastasis from gastric cancer. World J Gastrointest Oncol 10(6): 124-136, 2018. PMID:29988904. DOI: 10.4251/wjgo.v10.i6.124

18 Singh D, Arora R, Kaur P, Singh B, Mannan R and Arora S: Overexpression of hypoxia-inducible factor and metabolic pathways: possible targets of cancer. Cell Biosci 7: 62, 2017. PMID: 29158891. DOI: 10.1186/s13578-017-0190-2

19 Araujo EP, Carvalheira JB and Velloso LA: Disruption of metabolic pathways - perspectives for the treatment of cancer. Curr Cancer Drug Targets 6(1): 77-87, 2006. PMID: 16475977. DOI: $10.2174 / 156800906775471734$

20 Kahlert UD, Mooney SM, Natsumeda M, Steiger HJ and Maciaczyk J: Targeting cancer stem-like cells in glioblastoma and colorectal cancer through metabolic pathways. Int J Cancer 140(1): 10-22, 2017. PMID: 27389307. DOI: 10.1002/ijc.30259

21 West KA1, Castillo SS and Dennis PA: Activation of the PI3K/Akt pathway and chemotherapeutic resistance. Drug Resist Updat 5(6): 234-248, 2002. PMID: 12531180. DOI: 10.1016/ S1368-7646(02)00120-6

22 Oki E, Baba H, Tokunaga E, Nakamura T, Ueda N and Futatsugi M: Akt phosphorylation associates with LOH of PTEN and leads to chemoresistance for gastric cancer. Int J Cancer 117(3): 376380, 2005. PMID: 15900596. DOI: 10.1002/ijc. 21170

23 Raaijmakers JH and Bos JL: Specificity in Ras and Rap signaling. J Biol Chem 284(17): 10995-10999, 2009. PMID: 19091745. DOI: 10.1074/jbc.R800061200

24 Katagiri K, Maeda A, Shimonaka M and Kinashi T: RAPL, a Rap1-binding molecule that mediates Rap1-induced adhesion through spatial regulation of LFA-1. Nat Immunol 4(8): 741748, 2003. PMID: 12845325. DOI: $10.1038 /$ ni950

25 Shah S, Brock EJ, Ji K and Mattingly RR: Ras and Rap1: A tale of two GTPases. Semin Cancer Biol 54: 29-39, 2018. PMID: 29621614. DOI: 10.1016/j.semcancer.2018.03.005

Received February 2, 2019 Revised March 18, 2019 Accepted March 19, 2019 